\title{
Parte II: Monitorização Hemodinâmica Básica e Cateter de Artéria Pulmonar*
}

\section{Part II: Basic Hemodynamic Monitoring and the Use of Pulmonary Artery Catheter}

Fernando Suparregui Dias, Ederlon Rezende, Ciro Leite Mendes, Álvaro Réa-Neto, Cid Marcos David,

Guilherme Schettino, Suzana Margareth Ajeje Lobo, Alberto Barros, Eliézer Silva, Gilberto Friedman, José Luiz Gomes do Amaral, Marcelo Park, Maristela Monachini, Mirella Cristine de Oliveira, Murillo Santucci César Assunção, Nelson Akamine, Patrícia Veiga C Mello, Renata Andréa Pietro Pereira, Rubens Costa Filho,

Sebastião Araújo, Sérgio Félix Pinto, Sérgio Ferreira, Simone Mattoso Mitushima, Sydney Agareno,

Yuzeth Nóbrega de Assis Brilhante; Painel de Especialistas do Consenso Brasileiro de

Monitorização e Suporte Hemodinâmico da Associação de Medicina Intensiva Brasileira (AMIB).

\section{RESUMO}

JUSTIFICATIVA E OBJETIVOS: A monitorização de funções vitais é uma das mais importantes e essenciais ferramentas no manuseio de pacientes críticos na UTI. Hoje é possível detectar e analisar uma grande variedade de sinais fisiológicos através de diferentes técnicas, invasivas e não-invasivas. O intensivista deve ser capaz de selecionar e executar o método de monitorização mais apropriado de acordo com as necessidades individuais do paciente, considerando a relação risco-benefício da técnica. Apesar do rápido desenvolvimento de técnicas de monitorização não-invasiva, a monitorização hemodinâmica invasiva com o uso do cateter de artéria pulmonar (CAP) ainda é um dos procedimentos fundamentais em UTI. O objetivo destas recomendações é estabelecer diretrizes para o uso adequado dos métodos básicos de monitorização hemodinâmica e CAP.

MÉTODO: O processo de desenvolvimento de recomendações utilizou o método Delphi modificado para criar e quantificar o consenso entre os participantes. A AMIB determinou um coordenador para o consenso, o qual escolheu seis especialistas para comporem o comitê consultivo. Outros 18 peritos de diferentes regiões do país foram selecionados para completar o painel de 25 especialistas, médicos e enfermeiros. Um levantamento bibliográfico na MedLine de artigos na língua inglesa foi realizado no período de 1966 a 2004.

RESULTADOS: Foram apresentadas recomendações referentes a 55 questões sobre monitorização da pressão venosa central, pressão arterial invasiva e cateter de artéria pulmonar. Com relação ao CAP, além de re- comendações quanto ao uso correto foram discutidas as indicações em diferentes situações clínicas.

CONCLUSÕES: A avaliação da pressão venosa central e da pressão arterial, além das variáveis obtidas com o CAP permite o entendimento da fisiologia indispensável para o cuidado de pacientes graves. Entretanto, a correta utilização dessas ferramentas é fundamental para os possíveis benefícios decorrentes do uso.

Unitermos: Consenso, Cateter de Artéria Pulmonar, Monitorização Hemodinâmica, Recomendação.

\section{SUMMARY}

BACKGROUND AND OBJECTIVES: Monitoring of vital functions is one of the most important tools in the management of critically ill patients. Nowadays is possible to detect and analyze a great deal of physiologic data using a lot of invasive and non-invasive methods. The intensivist must be able to select and carry out the most appropriate monitoring technique according to the patient requirements and taking into account the benefit/risk ratio. Despite the fast development of non invasive monitoring techniques, invasive hemodynamic monitoring using Pulmonary Artery Catheter still is one of the basic procedures in Critical Care. The aim was to define recommendations about clinical utility of basic hemodynamic monitoring methods and the Use of Pulmonary Artery Catheter.

METHODS: Modified Delphi methodology was used to create and quantify the consensus between the participants. AMIB indicated a coordinator who invited more six experts in the area of monitoring and hemodynamic support to constitute the Consensus Advisory Board. 
Twenty-five physicians and nurses selected from different regions of the country completed the expert panel, which reviewed the pertinent bibliography listed at the MEDLINE in the period from 1996 to 2004.

RESULTS: Recommendations were made based on 55 questions about the use of central venous pressure, invasive arterial pressure, pulmonary artery catheter and its indications in different settings.

CONCLUSIONS: Evaluation of central venous pressure and invasive arterial pressure, besides variables obtained by the PAC allow the understanding of cardiovascular physiology that is of great value to the care of critically ill patients. However, the correct use of these tools is fundamental to achieve the benefits due to its use.

Key Words: Consensus, Hemodynamic Monitoring, Pulmonary Artery Catheter, Recommendation.

\section{INTRODUÇÃO}

A monitorização de funções vitais é uma das mais importantes e essenciais ferramentas no manuseio de pacientes críticos na UTI. Hoje, é possível detectar e analisar uma grande variedade de sinais fisiológicos através de diferentes técnicas, invasivas e não-invasivas. O intensivista deve ser capaz de selecionar e executar o método de monitorização mais apropriado de acordo com as necessidades individuais do paciente, considerando a relação risco-benefício da técnica. Apesar do rápido desenvolvimento de técnicas de monitorização não-invasiva, a monitorização hemodinâmica invasiva com o uso do cateter de artéria pulmonar ainda é um dos procedimentos fundamentais em UTI. O cateter de artéria pulmonar permite a monitorização de pressões na circulação pulmonar, do fluxo sangüíneo e da saturação venosa mista, além de fornecer dados para estimar o desempenho cardíaco e julgar a adequação do sistema cardiocirculatório. Informações obtidas de maneira cuidadosa e correta são a base para a apropriada avaliação hemodinâmica que habitualmente afeta a decisão terapêutica inicial.

\section{QUESTÃO: QUAIS SÃO AS VARIÁVEIS E MÉTODOS USADOS DURANTE A MONITORIZAÇÃO HEMODINÂMICA BÁSICA?}

Recomendação: As variáveis e métodos recomendados como componentes da Monitorização Hemodinâmica Básica são: freqüência cardíaca, diurese, ECG contínuo, $\mathrm{SpO}_{2}$, PAM não-invasiva, freqüência respiratória, temperatura, PVC e PAM invasiva. A utilização da monitorização com PAM invasiva deverá ser indicada de forma individualizada.

Grau D

Racional: A monitorização de sinais vitais é indispensável durante o acompanhamento de pacientes graves. Entretanto, cabe ressaltar que a sensibilidade destas variáveis é limitada, sendo tanto maior quanto mais grave o paciente. Portanto, se a normalização dos sinais vitais não é sinônimo de estabilidade, por outro lado, sinais vitais anormais são um importante alerta de gravidade ${ }^{1}$.

\section{QUESTÃO: TODO O PACIENTE COM INSTABILIDADE HEMODINÂMICA DEVE SER MONITORIZADO COM PRESSÃO VENOSA CENTRAL (PVC)?}

Recomendação: Sim. A medida da PVC é um dos primeiros passos na avaliação da volemia e da função cardíaca de pacientes graves.

Grau B1.

Racional: A PVC pode contribuir para avaliação e controle do estado do volume intravascular. Como tal análise relaciona retorno venoso com função ventricular, variações na complacência de um dos ventrículos ou na capacitância venosa podem provocar erros nesta correlação, como ocorre na disfunção diastólica, na ventilação mecânica e com o uso de drogas vasoativas. A avaliação da PVC como indicador de volemia deve ser realizada através da resposta à infusão de líquidos, de modo seriado. A ausência de aumentos na PVC de até $3 \mathrm{mmHg}$, após prova de volume padronizada, quase sempre é garantia de bom desempenho cardíaco e de espaço para reposição volêmica. Apesar das limitações como método de avaliação da volemia, é o mais simples, pouco invasivo e disponível rapidamente à beira do leito. Valores extremos de PVC, isto é, muito baixos ou muito elevados, correspondem a hipovolemia e hipervolemia, respectivamente. Além disso, a PVC pode ser utilizada como um dos parâmetros de otimização hemodinâmica, como já demonstrado em pacientes sépticos graves. A variação de 2 a $3 \mathrm{mmHg}$ durante a inspiração sugere que este grupo de pacientes tem maior probabilidade de aumentar o índice cardíaco em resposta à infusão de líquidos. A PVC deve ser utilizada em todo paciente no qual haja dúvida quanto ao estado volêmico e cuja correção interfira na evolução clínica, principalmente nos estágios iniciais das seguintes condições: a) choque de qualquer etiologia; b) desconforto respiratório 
grave; c) insuficiência renal aguda; d) sepse grave; e e) aciente com alto risco cirúrgico e submetido à cirurgia de grande porte ${ }^{2-4}$.

\section{QUESTÃO: QUEM NÃO DEVE SER MONITORIZADO COM PVC?}

Recomendação: Não há contra-indicação à monitorização da PVC, mas sim ao acesso vascular central.

Grau D

Racional: As contra-indicações são ao acesso vascular central: pacientes com síndromes obstrutivas da veia cava superior, trombose venosa profunda de membros superiores, infecção ou queimadura nos locais de acesso, bem como limitações anatômicas. Entretanto, o acesso venoso central, na maioria dos pacientes graves, é factível e realizado sem dificuldades ${ }^{5}$.

\section{QUESTÃO: QUAL A TÉCNICA MAIS ADEQUADA PARA MEDIDA DA PVC?}

Recomendação: Deve-se preferir a monitorização eletrônica contínua, utilizando-se transdutores de pressão. Grau B2

Racional: A medida da pressão venosa central através de manômetro ou coluna líquida é menos precisa do que a medida eletrônica contínua com a utilização de transdutores de pressão. Esta apresenta melhor correlação com a medida através do registro do traçado de pressão venosa, considerado "padrão ouro". Quando a monitorização digital contínua não for possível, a precisão de medidas manométricas parece melhor quando são utilizadas as veias jugulares internas ou a subclávia esquerda ${ }^{4,6}$.

\section{QUESTÃO: QUAIS CUIDADOS DEVEM SER TOMADOS DURANTE A VERIFICAÇÃO DA PVC?}

Recomendações: Os cuidados são, na seqüência:

1. Manter o paciente em posição supina, sem o travesseiro;

2. Certificar-se do correto posicionamento do transdutor;

3. Identificar o zero hidrostático

4. Verificar o comprimento do circuito (até $110 \mathrm{~cm}$ );

5. Verificar o preenchimento completo do cateter com líquido (remover bolhas e coágulos);

6. Realizar o Teste de "lavagem" ("Flush Test");

7. "Zerar" o sistema em relação à pressão atmosférica;

8. Verificar o posicionamento da ponta do cateter venoso central;
9. Identificar as ondas de PVC;

10. Localizar o componente "a" da PVC/PAD, visto que é o correspondente à contração atrial, e em seguida localizar o ponto imediatamente anterior a "c", ou seja, aquele que ocorre antes do fechamento da valva tricúspide, que corresponde à fase final da diástole e onde os ventrículos se encontram praticamente preenchidos, gerando a pressão diastólica final, que é a pressão a ser medida. Procurar relacionar a onda "a" do traçado pressórico com o final da onda $\mathrm{P}$ do traçado do ECG e a onda "c" com o ponto "z", que identifica o final do complexo QRS. Caso o monitor não disponha da tecnologia para congelar as ondas de pressão e o traçado de ECG, levar em consideração a média da PVC durante a fase expiratória do ciclo respiratório.

11. Realizar a medida no final da expiração, tanto em pacientes intubados como em ventilação espontânea; 12. Verificar a morfologia da curva (afastar sub ou superamortecimento) e sua relação com o ciclo respiratório.

Grau D

Racional: Apesar da precisão dos equipamentos eletrônicos e da grande vantagem de medir continuamente a pressão, o "padrão ouro" para a medida de pressões exige o registro gráfico da curva e sua análise minuciosa, sem esquecer dos fatores que podem comprometer a precisão, já descritos ${ }^{7-9}$.

\section{QUESTÃO: QUAL O RISCO DO USO DE UM CATETER VENOSO CENTRAL (CVC) PARA MONITORIZAÇÃO HEMODINÂMICA PELA PVC?}

Recomendação: Este é um procedimento de baixo risco ${ }^{10}$

Grau D

Racional: Os CVC são indispensáveis na prática médica moderna, principalmente em UTI, onde o seu uso tem sido crescente. Esses dispositivos são utilizados principalmente em pacientes idosos, portadores de co-morbidades, com hospitalização prolongada e em uso de antibióticos de largo espectro. Tais fatores aumentam a incidência e gravidade de complicações mecânicas e infecciosas relacionadas ao uso dos CVC. A melhoria, tanto na habilidade dos médicos durante o procedimento de inserção dos cateteres quanto dos cuidados de enfermagem durante a sua permanência, tem contribuído para reduzir as taxas de complicações associadas ao uso de tais dispositivos ${ }^{11}$. 


\section{QUESTÃO: O PACIENTE DEVE SER RETIRADO DA VENTILAÇÃO MECÂNICA PARA A MEDIDA DA PVC?}

Recomendação: Não. O desrecrutamento alveolar promovido por esta manobra, como se sabe, é extremamente deletério para o paciente.

Grau C

Racional: Embora durante a ventilação mecânica ocorra aumento da pressão intratorácica, elevando artificialmente a PVC, especialmente quando se utiliza alto nível de PEEP, a retirada do paciente da ventilação mecânica não é recomendada. A interrupção da ventilação, e em especial da PEEP, pode causar efeitos deletérios $^{12}$. É necessário ter conhecimento de que a ventilação mecânica com pressão positiva pode determinar interferência no valor absoluto da PVC e levar isso em consideração ao tomar condutas clínicas baseadas no seu valor. Deve-se, nesta circunstância, considerar que os valores de PVC estejam superestimados. O mais importante, porém, é a avaliação contínua da PVC e da sua tendência.

\section{QUESTÃO: QUAL A VIA PREFERENCIAL DE CATETERIZAÇÃO VENOSA CENTRAL PARA MONITORIZAÇÃO DA PVC?}

Recomendação: Em ordem de preferência:

1. Jugular direita;

2. Jugular esquerda;

3. Subclávia esquerda;

4. Subclávia direita;

5. Femoral direita ou esquerda; e

6. PICC.

Grau B2.

Racional: A punção da veia jugular acompanha-se de menor incidência de complicações mecânicas, porém maior incidência de complicações infecciosas, quando comparada ao sítio da veia subclávia ${ }^{13}$.

\section{QUESTÃO: QUAL A REFERÊNCIA ANATÔMICA PARA DEFINIR O ZERO HIDROSTÁTICO?}

Recomendação: No quarto espaço intercostal, à altura da linha axilar média (ponto médio entre a parede anterior e posterior do tórax).

Grau C

Racional: Esse ponto corresponde ao eixo flebostático, que se encontra à altura do AD. É importante lembrar que a utilização deste ponto pode superestimar a PVC real em até $5 \mathrm{mmHg}^{14,15}$.

\section{QUESTÃO: QUAL DEVE SER O TEMPO DE PERMANÊNCIA MÁXIMO DO CATETER VENOSO CENTRAL PARA MEDIDA DA PVC?}

Recomendação: Não há limite definido, devendo o tempo ser o mínimo necessário.

Grau D

Racional: O cateter venoso central deve ser retirado o mais precocemente possível, desde que o paciente não mais necessite de avaliação da PVC ou administração de drogas por via central. Se houver eritema local estendendo-se além de quatro milímetros, ao redor do ponto de sua inserção, purulência e sinais inflamatórios locais francos ou sinais de sepse relacionada ao cateter, culturas devem ser obtidas. Nova punção deve ser realizada em outro sítio se um cateter removido com fio guia apresentar mais de quinze UFC em cultura semiquantitativa. Trocar om fio guia, se foi colocado em situação de emergência, sem assepsia adequada. O bom funcionamento do cateter também determina o seu tempo de permanência. Cateteres com problemas de infusão ou refluxo devem ser trocados, pois levam à falta de acurácia na aferição da PVC ${ }^{16-18}$.

\section{QUESTÃO: QUAL DEVE SER O POSICIONAMENTO ADEQUADO DA EXTREMIDADE DISTAL DO CVC?}

Recomendação: A localização ideal da ponta do cateter é na região distal da veia inominada ou proximal da veia cava superior, longe da parede e paralelo ao longo de seu eixo. Grau D

Racional: A medida da PVC neste posicionamento reflete com precisão aceitável a pressão no interior do átrio direito e evita a movimentação do cateter no interior do átrio e sua migração para o ventrículo direito, com o conseqüente risco de perfuração dessa câmara e tamponamento cardíaco, que é a complicação mecânica mais temível nessa circunstância ${ }^{11}$.

\section{QUESTÃO: O CONTROLE RADIOLÓGICO DO POSICIONAMENTO DO CATETER DEVE SER OBRIGATÓRIO?}

Recomendação: Após o procedimento, todos os pacientes devem ser radiografados.

Grau D

Racional: O controle radiológico é uma técnica segura para se conhecer a localização exata da ponta do cateter e avaliar se houve complicações imediatas relacionadas à punção venosa central ${ }^{19}$. 


\section{QUESTÃO: DE QUE FORMA SE DEVE INTERPRETAR A PVC COMO INDICADOR DE VOLEMIA?}

Recomendação: Considerando a sua variação em resposta a testes de volume como referência e associadamente a outros parâmetros hemodinâmicos e de perfusão.

Grau B3

Racional: Os níveis baixos normalmente correspondem a hipovolemia; os níveis normais ou elevados não têm boa correlação com o estado volêmico. Em pacientes sob ventilação mecânica e PEEP elevada, a PVC pode não refletir de forma adequada a volemia do paciente. O mesmo pode ocorrer em pacientes com insuficiência tricúspide ou com áreas hipocinéticas ou discinéticas e Fração de Ejeção de Ventrículo Esquerdo (FEVE) menor que $50 \%{ }^{2,20}$.

\section{QUESTÃO: TODO PACIENTE GRAVE COM INSTABILIDADE HEMODINÂMICA DEVE SER MONITORADO COM PRESSÃO ARTERIAL MÉDIA (PAM) INVASIVA?}

Recomendação: Sim, visto que um dos principais objetivos durante o suporte hemodinâmico é manter PAM em nível suficiente para garantir adequada perfusão tecidual. Grau C

Racional: Uma vez que pode haver erros importantes quando se verifica a pressão arterial por métodos nãoinvasivos, principalmente nos pacientes em uso de drogas vasoativas ou com instabilidade hemodinâmica grave, deve-se optar pela medida invasiva da PAM, que permite a avaliação de forma contínua e mais precisa dos níveis pressóricos ${ }^{21}$.

\section{QUESTÃO: QUEM DEVE SER MONITORADO COM A PAM INVASIVA?}

Recomendação: Os pacientes em emergências hipertensivas, estados de choque, em uso de aminas vasoativas, vasodilatadores, vasopressores ou inotrópicos. Outra indicação é a necessidade da obtenção freqüente de amostras de sangue para gasometria, como no paciente com insuficiência respiratória e grave anormalidades do equilíbrio ácido-base. Ainda deve ser realizada em pacientes em intra e pós-operatório imediato de cirurgia cardíaca e neurológica ou outras condições nas quais não se pode tolerar hipotensão ou variações bruscas da PAM, como durante a monitorização da PIC e em pacientes em uso de balão intra-aórtico.

Grau C

Racional: A monitorização invasiva da pressão arterial tem maior acurácia que a medida não invasiva nas situações já citadas ${ }^{21}$.

\section{QUESTÃO: QUAIS AS CONTRA-INDICAÇÕES PARA UTILIZAÇÃO DE CATETER ARTERIAL DURANTE A MONITORAÇÃO COM A PAM?}

Recomendação: Não há contra-indicação absoluta.

Grau D

Racional: As contra-indicações são aquelas relativas à punção arterial:

1. Doença vascular periférica;

2. Doenças hemorrágicas ou uso de anticoagulantes e trombolíticos;

3. Áreas infectadas; e

4. Queimaduras nos locais de punção²2.

\section{QUESTÃO: QUAIS TÉCNICAS DEVEM SER UTILIZADAS PARA ACESSO DO CATETER ARTERIAL E AFERIÇÃO DA PAM INVASIVA?}

Recomendação: Contínua e com transdutor de pressão. O acesso deve ser realizado por punção. Quando não for possível, efetuar dissecção.

Grau D.

Racional: A medida invasiva da pressão arterial através de manômetro é menos precisa do que a contínua e eletrônica, com a utilização de transdutores de pressão. Esta última apresenta melhor correlação com a medida através do registro do traçado de pressão arterial, considerado "padrão ouro" ${ }^{23}$. A utilização de coluna de mercúrio em sistema aberto não é permitida pela Agência Nacional de Vigilância Sanitária (Resolução RE n 16, de 06 de julho de 2004). A grande evolução da cardiologia intervencionista trouxe relevante progresso às técnicas de punção arterial percutânea, procedimento mais simples e seguro do que a dissecção ${ }^{24}$.

\section{QUESTÃO: QUAIS CUIDADOS DEVEM SER TOMADOS NA MONITORIZAÇÃO HEMODINÂMICA, COM A UTILIZAÇÃO DE PAM INVASIVA?}

Recomendação: Usar material adequado e observar a técnica correta. Na ordem, os cuidados devem ser: 
1. Realizar manobra de Allen;

2. Usar material adequado para punção arterial;

3. Verificar zero hidrostático;

4. "Zerar" o sistema em relação à pressão atmosférica

5. Procurar e reparar vazamentos e bolhas;

6. Analisar a morfologia da curva (para descartar sub e superamortecimento);

7. Determinar a resposta dinâmica do sistema de mensuração através do "teste de lavagem" ("fast flush");

8. Limitar o comprimento dos equipos;

9. Observar conexões e extensões;

10. Manter o posicionamento neutro do membro onde está inserido o cateter;

11. Fixar adequadamente o sistema;

12. Posicionar cateter e transdutor;

13. Manter cuidados com o local de inserção do cateter, avaliando-se sinais de flogose, sangramento, perfusão periférica, presença de trombos ou obstáculo no cateter;

14. Atentar para os riscos de complicações: embolização arterial e sistêmica, insuficiência vascular, necrose, isquemia, infecções, hemorragias, injeção acidental de drogas por via intra-arterial, trombose, espasmos arteriais, hematoma local, dor local, fístula artériovenosa;

15. Manter o cateter o mínimo de tempo necessário.

Grau D

Racional: O cumprimento destes cuidados, além de garantir a precisão do método, contribui para reduzir o risco de complicações, que apesar de baixo, não deve ser negligenciado. Dentre as principais, destacam-se: embolização arterial e sistêmica, insuficiência vascular, necrose, isquemia, infecções, hemorragias, injeção acidental de drogas por via intra-arterial, trombose, espasmos arteriais, hematoma e dor locais e fístula arteriovenosa ${ }^{25}$.

\section{QUESTÃO: NA CATETERIZAÇÃO ARTERIAL PARA MONITORIZAÇÃO DA PAM, QUAL DEVE SER O ACESSO PREFERENCIAL?}

Recomendação: A primeira escolha é a artéria radial, seguida da artéria femoral.

Grau C.

Racional: Em pacientes cirúrgicos sem instabilidade hemodinâmica, o acesso de preferência é a artéria radial. Em pacientes críticos com instabilidade hemodinâmica, a via preferida é a artéria femoral, por ser menos propensa a fenômenos vasomotores ${ }^{26}$.

\section{QUESTÃO: O TESTE DE ALLEN DEVE SER REALIZADO NO CASO DE SE OPTAR PELA UTILIZAÇÃO DA ARTÉRIA RADIAL?}

Recomendação: Sim, a integridade da arcada palmar deve ser avaliada antes do procedimento ainda que de forma subjetiva.

Grau C.

Racional: Embora sem a precisão de outros métodos (Doppler e pletismografia), é uma manobra de fácil execução à beira do leito ${ }^{27}$.

\section{QUESTÃO: DURANTE A MONITORIZAÇÃO DA PAM, POR QUANTO TEMPO É SEGURO MANTER O CATETER?}

Recomendação: Não há limite definido, sendo o tempo o mínimo necessário.

Grau C

Racional: O cateter de PAM deve ser retirado o mais precocemente possível, desde que o paciente não mais necessite da punção arterial. Deve ser retirado ou trocado quando ocorrerem complicações decorrentes de seu uso ${ }^{28}$.

\section{QUESTÃO: PACIENTES INSTÁVEIS HEMODINAMICAMENTE E AQUELES COMPENSADOS HEMODINAMICAMENTE, MAS COM RISCO DE DESCOMPENSAÇÃO, DEVEM SER MONITORIZADOS COM CATETER DE ARTÉRIA PULMONAR (CAP)?}

Recomendação: A indicação do CAP nestes grupos é incerta.

Grau: Vide subgrupos específicos.

Racional: Alguns subgrupos beneficiam-se da utilização do CAP:

1. Pacientes cirúrgicos: $O$ uso do CAP deveria ser considerado em pacientes de alto risco e com trauma grave. O risco de alteração hemodinâmica deve considerar três variáveis: as co-morbidades prévias, o tipo de cirurgia e a habilitação técnica da equipe;

2. Pacientes clínicos: Em pacientes com IAM complicado com disfunção ventricular grave ou suspeita de complicação mecânica, ICC grave, choque de qualquer etiologia e pacientes com SDRA ${ }^{29,30}$.

\section{QUESTÃO: O CAP PODE AJUDAR NA AVALIAÇÃO DE QUAIS PARÂMETROS?}

Recomendações: 
O CAP serve para dirigir:

1. Uso de inotrópicos;

2. Reposição volêmica;

3. Uso de drogas vasopressoras; e avaliar:

4. Pressões de enchimento;

5. Função miocárdica;

6. Adequação da oxigenação tecidual.

Grau D.

Racional: O CAP propicia informações que não podem ser aferidas de forma precisa apenas com a avaliação clínica ou hemodinâmica básica ${ }^{31,32}$.

\section{QUESTÃO: POR QUE E QUANDO MONITORIZAR COM CAP O PACIENTE COM SÍNDROME DO DESCONFORTO RESPIRATÓRIO AGUDO (SDRA)?}

Recomendações: O CAP deve ser especialmente considerado nas seguintes circunstâncias:

1) Emprego de níveis elevados de pressão em vias aéreas;

2) Insuficiência renal aguda concomitante;

3) Piora progressiva de parâmetros de perfusão tecidual;

4) Durante procedimentos que possam deteriorar o estado hemodinâmico, como hemodiálise e cirurgia;

5) Disfunção ventricular esquerda associada ao quadro;

6) Hipertensão arterial pulmonar com grave disfunção de ventrículo direito.

Grau D

Racional: Na SDRA, pequenos e rápidos aumentos da POAP pioram o edema em decorrência do aumento da permeabilidade capilar pulmonar. A manutenção de níveis mais baixos de pressão poderia minimizar a transudação de líquidos. Contudo, se a POAP é muito baixa, pode haver prejuízo para o fluxo sangüíneo em diferentes órgãos, facilitando a evolução de disfunções. Deve-se assumir que o uso do CAP permite a orientação mais segura da quantidade de fluidos e da necessidade de inotrópicos, determinando um controle mais preciso da terapêutica $^{33,34}$.

\section{QUESTÃO: POR QUE E QUANDO O PACIENTE COM EDEMA PULMONAR DE ETIOLOGIA INDEFINIDA DEVE SER MONITORIZADO COM CAP?}

Recomendação: Sempre que houver dúvida diagnóstica a respeito da etiologia do edema pulmonar, o CAP pode auxiliar na diferenciação entre o de origem cardiogênica e aquele não cardiogênico, além de, obviamente, orientar a terapia.

Grau C

Racional: A medida da POAP auxilia na identificação do edema pulmonar por estimar o valor da pressão hidrostática, enquanto a medida do Débito Cardíaco estima a função ventricular esquerda ${ }^{32}$.

\section{QUESTÃO: DEVEMOS MONITORIZAR COM CAP O PACIENTE EM SEPSE GRAVE?}

Recomendação: Excepcionalmente, apenas quando houver progressão da disfunção orgânica, apesar da obediência a metas terapêuticas estabelecidas para essa condição ${ }^{3}$.

Grau B1

Racional: Na sepse grave, a hipoperfusão tecidual caracterizada por hiperlactatemia ou saturação venosa de oxigênio baixa indica a necessidade de reanimação hemodinâmica guiada por metas pré-estabelecidas. Nas primeiras seis horas de reanimação devemos ter como objetivos: manter uma PVC entre 8 e $12 \mathrm{mmHg}$, uma PAM maior que $65 \mathrm{mmHg}$ e a $\mathrm{SvcO}_{2}$ maior ou igual a $70 \%^{3,35}$.

\section{QUESTÃO: DEVEMOS MONITORIZAR COM CAP O PACIENTE COM CHOQUE SÉPTICO?}

Recomendação: Sim. Para guiar a terapêutica e tentar evitar a progressão de disfunções orgânicas, principalmente quando não há resposta satisfatória à reanimação agressiva com fluídos.

Grau B3

Racional: Na sepse, a presença de disfunção cardiovascular associa-se a um pior prognóstico. No choque séptico, a dependência do consumo em relação à oferta de oxigênio é mais ampla, em comparação a outros estados de choque, e por esta razão uma condição hiperdinâmica habitualmente se faz necessária para atender a demanda do paciente. A utilização do CAP fornece informações que permitem otimizar a reposição de volume e drogas vasoativas, visando à restauração das variáveis de perfusão tecidual. Em um hospital universitário americano, a comparação de duas coortes consecutivas de um ano demonstrou uma menor mortalidade quando se utilizou, com maior freqüência, CAP durante o suporte hemodinâmico de pacientes com choque séptico ${ }^{36-39}$. 


\section{QUESTÃO: DEVEMOS MONITORIZAR COM CAP O PACIENTE COM IAM E CHOQUE CARDIOGÊNICO?}

Recomendação: Sim. A monitorização com o CAP permite um acompanhamento mais rigoroso desses pacientes, facilitando o diagnóstico e otimização da reposição de volume, uso de drogas vasoativas e o emprego de assistência circulatória mecânica visando à restauração das variáveis de perfusão tecidual. Auxilia ainda no diagnóstico de complicações mecânicas associadas ao IAM, freqüentes nesses pacientes.

Grau B2

Racional: O manuseio de pacientes na fase aguda do infarto com choque cardiogênico guiado pelo CAP pode ser benéfico. No GUSTO-I, os pacientes com IAM da coorte americana do estudo foram manejados de modo mais agressivo, incluindo uso mais freqüente do CAP que em outros países e apresentaram uma melhor evolução em acompanhamento de um ano. Contudo, uma vez que a abordagem mais agressiva incluiu além do uso mais freqüente do CAP também indicações mais amplas e precoces de angioplastia percutânea e cirurgia de revascularização miocárdica, não é possíveis afirmar que o uso do CAP foi responsável pela menor mortalidade. Entretanto, na opinião de diversos especialistas, merece destaque a relação entre tratamento precoce e desfecho e, nesse contexto, a dificuldade em fazer o diagnóstico clínico de choque cardiogênico é uma importante justificativa para a utilização do CAP nesses pacientes. Não se dispõe de algoritmos para o tratamento nesse grupo com os dados obtidos com o CAP, entretanto, observou-se no estudo GUSTO-I que os pacientes com débito cardíaco de 5,1 L/min e POAP de $20 \mathrm{mmHg}$ apresentaram menor mortalidade do que os pacientes com condição hemodinâmica diferente. Outros estudos, todavia, não conseguiram demonstrar benefícios com a utilização do CAP no manejo destes pacientes, porém, além do fator de confusão de estudos observacionais, onde o CAP é empregando nos pacientes mais graves, a falta de recomendações para intervenções terapêuticas precisas com as informações obtidas a partir deste método de monitorização dificulta a interpretação desses resultados $29,40-42$.

\section{QUESTÃO: POR QUE E QUANDO MONITORIZAR O PACIENTE COM CHOQUE DE QUALQUER ETIOLOGIA?}

Recomendação: Para otimizar a reposição de volume e drogas vasoativas, visando à restauração das variáveis de perfusão tecidual, sempre o mais precocemente possível43.

Grau D.

Racional: Mimoz e col. ${ }^{44}$ estudaram o impacto do CAP em pacientes com choque não responsivo à infusão de fluidos e sem IAM. A mortalidade foi de $100 \%$ no subgrupo manejado sem CAP e 59\% nos pacientes que tiveram sua terapia modificada em função da sua utilização.

\section{QUESTÃO: PACIENTES COM PRÉ-ECLAMPSIA DEVEM SER MONITORIZADOS COM CAP?}

Recomendação: Não há recomendação para uso do CAP neste subgrupo de pacientes.

Grau D.

Racional: O uso do CAP não é recomendado rotineiramente neste subgrupo, pois não há estudos controlados e aleatórios justificando a utilidade clinica do CAP ${ }^{45}$.

\section{QUESTÃO: OS PACIENTES CIRÚRGICOS DE ALTO RISCO DEVEM SER MONITORIZADOS COM CAP NO PERI-OPERATÓRIO?}

Recomendação: Sim. Porque a otimização peri-operatória de parâmetros de oxigenação e perfusão, através de protocolos rígidos, diminui a mortalidade dessa população. O CAP deve ser inserido anteriormente ao início da cirurgia.

Grau A1

Racional: Há diversos estudos de otimização hemodinâmica pré-operatória com o objetivo de tornar ótimos os parâmetros hemodinâmicos e de perfusão tecidual, que demonstraram redução na mortalidade ${ }^{46-48}$.

\section{QUESTÃO: ESTÁ INDICADO O USO DO CAP NO PACIENTE NEUROCRÍTICO?}

Recomendação: Não há recomendação para uso do CAP neste subgrupo de pacientes.

Grau D

Racional: Não há estudos controlados e aleatórios avaliando a utilidade clinica do CAP para justificar seu uso rotineiro nesses pacientes ${ }^{39,49,50}$.

\section{QUESTÃO: O PACIENTE SUBMETIDO A TRANSPLANTE DE ÓRGÃO SÓLIDO DEVE SER MONITORIZADO COM CAP?}

Recomendação: Não há indicação para o uso rotineiro, mas apenas em casos selecionados, como nos de 
alta complexidade, como fígado, coração e pulmão e sempre precocemente, antes do desenvolvimento de disfunção orgânica ${ }^{51}$.

Grau D

Racional: Não há estudos controlados e aleatórios avaliando a utilidade clinica do CAP para justificar o uso rotineiro nessas pacientes.

\section{QUESTÃO: QUANDO MONITORIZAR COM CAP O PACIENTE SUBMETIDO À CIRURGIA CARDÍACA?}

Recomendação: O CAP não é recomendado para uso rotineiro nesse subgrupo de pacientes.

Grau D

Racional: Não há evidência de benefício do uso do CAP de forma sistemática em pacientes de cirurgia cardíaca. Está indicado em procedimentos complicados e cirurgia de alto risco de instabilidade hemodinâmica, como em pacientes com disfunção ventricular (FEVE < 40\%) ou valvular grave, além de comprometimento importante em outros órgãos (pulmões e rins) $)^{52,53}$.

\section{QUESTÃO: POR QUE MONITORIZAR COM CAP O PACIENTE DOADOR DE ÓRGÃO?}

Recomendação: O CAP não está recomendado para uso rotineiro nesse subgrupo de pacientes.

Grau D

Racional: Não há estudos controlados e aleatórios avaliando a utilidade clinica do CAP para justificar seu uso rotineiro nesse subgrupo ${ }^{54,55}$.

\section{QUESTÃO: DEVEMOS MONITORIZAR COM CAP O PACIENTE COM POLITRAUMA GRAVE?}

Recomendação: Sim. Alguns estudos sugerem que a otimização hemodinâmica precoce através de protocolos rígidos de tratamento diminui a mortalidade desses pacientes.

Grau B1

Racional: Há diversos estudos de otimização hemodinâmica pré-operatória com o objetivo de tornar ótimos os parâmetros hemodinâmicos e de perfusão tecidual que demonstraram redução na mortalidade nesse subgrupo ${ }^{56-58}$. Estudo aleatório conduzido em grandes queimados demonstrou que a reanimação guiada por metas obtidas com CAP, resultou em melhor adequação da volemia em comparação a reanimação guiada pela fórmula de Baxter ${ }^{59}$.

\section{QUESTÃO: O PACIENTE COM DISFUNÇÃO AGUDA DE VENTRÍCULO DIREITO DEVE SER MONITORIZADO COM CAP?}

Recomendação: Sim. O diagnóstico e acompanhamento de pacientes com disfunção aguda de ventrículo direito à beira do leito continuam um grande desafio e a termodiluição é um dos poucos métodos que permitem o acompanhamento contínuo.

Grau D

Racional: Deve-se monitorizar com o CAP quando não houver resposta imediata à reposição volêmica e às drogas vasoativas. Sempre que possível, utilizar o CAP volumétrico, que permite a medida da fração de ejeção e do volume diastólico final do ventrículo direito ${ }^{60,61}$.

\section{QUESTÃO: E QUEM NÃO DEVE SER MONITORIZADO COM CAP (CONTRA- INDICAÇÕES)?}

Recomendações: Pacientes em fase terminal ou com determinação de limite para o suporte terapêutico, com risco inaceitável para acesso venoso e na presença de hipotermia.

Grau D

Racional: O uso de tecnologia avançada para pacientes em fase terminal constitui-se em futilidade; na presença de hipotermia há risco aumentado de disritmias cardíacas ${ }^{62}$.

\section{QUESTÃO: QUAIS CUIDADOS DEVEM SER TOMADOS NA MONITORIZAÇÃO HEMODINÂMICA COM A UTILIZAÇÃO DO CAP?}

Recomendações: Os cuidados são, em seqüência: 1. Manter o paciente em posição supina, sem o travesseiro;

2. Certificar-se do correto posicionamento do transdutor em relação ao decúbito;

3. Identificar o zero hidrostático

4. Verificar comprimento do circuito (até $110 \mathrm{~cm}$ );

5. Examinar o preenchimento completo do cateter com líquido (remover bolhas e coágulos);

6. Realizar Teste de "Lavagem" ("Flush Test");

7. "Zerar" com a pressão atmosférica;

8. Identificar as ondas de CAP: PAD, PVD, PAP e POAP;

9. Procurar relacionar a onda "a" do traçado pressórico com o final da onda P do traçado do ECG;

10. Realizar a medida no final da expiração, tanto em pacientes intubados como naqueles com ventilação espontânea; 
11. Verificar a morfologia das curvas (para afastar sub e superamortecimento) e suas relações com o ciclo respiratório;

12. Verificar o posicionamento da ponta do cateter através de radiografia de tórax;

Grau D

Racional: Informações obtidas de maneira cuidadosa e corretas são a base para a apropriada avaliação hemodinâmica que habitualmente afeta a decisão terapêutica inicial ${ }^{7}$.

\section{QUESTÃO: QUAL O RISCO DA MONITORIZAÇÃO HEMODINÂMICA PELO CAP?}

Recomendação: O risco da monitorização hemodinâmica com CAP é considerado baixo.

Grau C

Racional: Vários estudos não conseguiram demonstrar associação entre CAP e mortalidade e a incidência de complicações é relativamente baixa ${ }^{63,64}$.

\section{QUESTÃO: NA MONITORIZAÇÃO HEMODINÂMICA COM O CAP, POR QUANTO TEMPO É SEGURO MANTER O CATETER?}

Recomendação: Não há limite definido e o tempo deve ser o mínimo necessário.

Grau C

Racional: Dependendo da necessidade de orientação diagnóstica e terapêutica, deve-se restringir o uso do CAP ao menor tempo possível (menos de 96 horas). Após este período, é recomendável reinserir outro cateter, se a monitorização hemodinâmica com o CAP ainda se fizer necessária, pois as complicações aumentam substancialmente ${ }^{64,65}$.

\section{QUESTÃO: QUANTO À APLICAÇÃO CLÍNICA DOS DIFERENTES TIPOS DE CATETERES DE ARTÉRIA PULMONAR, ESPECIFIQUE O NÍVEL DE IMPORTÂNCIA:}

Recomendação: O emprego de CAP com $\mathrm{SvO}_{2}$ é considerado muito importante; o do CAP com DC contínuo e CAP volumétrico com DC contínuo é considerado importante $e^{65-68}$.

Grau D

\section{QUESTÃO: EM QUAIS SITUAÇÕES CLÍNICAS O CAP VOLUMÉTRICO DEVE SER UTILIZADO?}

Recomendação: O CAP volumétrico deve ser utilizado nas seguintes situações: disfunção aguda de VD; politrauma; hipertensão intra-abdominal; ventilação mecânica com altas pressões na via aérea; e como auxiliar para o diagnóstico diferencial entre IAM de VD e tamponamento cardíaco. Grau C

Racional: Nessas situações, ocorre alteração na complacência dos ventrículos, sendo mais preciso estimar a pré-carga através de variáveis volumétricas ${ }^{66}$.

\section{QUESTÃO: EM QUAIS SITUAÇÕES CLÍNICAS O CAP COM MEDIDA CONTÍNUA DE DÉBITO CARDÍACO DEVE SER UTILIZADO?}

Recomendação: Sempre que possível, deve-se optar pela utilização do CAP com medida contínua de débito cardíaco.

Grau D

Racional: Em todas as situações com indicação de CAP, a monitorização contínua do DC com curva de tendência possibilita intervenções precoces, por que permitem identificar de forma mais rápida as alterações relacionadas à função cardiovascular ${ }^{6}$.

\section{QUESTÃO: EM QUAIS SITUAÇÕES CLÍNICAS O CAP COM MEDIDA DA SVO ${ }_{2}$ DEVE SER UTILIZADO?}

Recomendação: Sempre que possível.

Grau D

Racional: $\mathrm{A} \mathrm{SvO}_{2}$ é indicador "padrão ouro" do balanço entre oferta e consumo global de oxigênio e tem relação fisiológica estreita com o DC. $\mathrm{SvO}_{2}$ baixa é fortemente indicativa de falta de adequação do $\mathrm{DC}$. $\mathrm{SvO}_{2}$ normal, por sua vez, não garante adequação da oferta à demanda de oxigênio dos tecidos ${ }^{69}$.

\section{QUESTÃO: QUAL O NÚMERO MÍNIMO RECOMENDÁVEL DE CAP INSERIDOS PARA HABILITAR O PROFISSIONAL DE MEDICINA INTENSIVA A ESTE MÉTODO?}

Recomendação: 25 para habilitação e 10 por ano para manter a qualificação.

Grau D

Racional: Devido ao risco de complicações, é de fundamental importância que a monitorização invasiva seja realizada por médicos experientes com a técnica de inserção para viabilizar o procedimento com segurança e com nível de conhecimento avançado para a correta averiguação e interpretação dos dados obtidos. Essa 
habilidade técnica é cognitiva, e uma vez adquirida, deve ser praticada com uma freqüência mínima para que não haja perda dessa capacidade. A competência na introdução do cateter está relacionada à experiência, à qualidade do treinamento inicial e à prática regular do procedimento após a habilitação ${ }^{29,70}$.

\section{QUESTÃO: O CAP PODE REDUZIR MORTALIDADE, MORBIDADE, CUSTOS E TEMPO DE INTERNAÇÃO?}

Recomendação: Incerto. O CAP é um instrumento de auxilio diagnóstico e não terapêutico per se.

Grau D

Racional: O benefício com o emprego do CAP dependerá em larga escala da qualificação dos profissionais em indicar a situação adequada para o seu uso, em interpretar adequadamente os dados obtidos e em tomar a decisão clinica mais acertada para a situação. Portanto, é difícil realizar estudos que avaliem a relação direta do uso do CAP com mortalidade, morbidade, custos e internação. A literatura existente é passível de muitas críticas metodológicas e é, desta forma, controversa. Existem estudos sugerindo aumento da mortalidade e tempo de internação sem, no entanto, permitir justificar diretamente esse achado ao uso do CAP. Paralelamente, há outros estudos que correlacionam o uso do CAP com melhor prognóstico e outros que não mostram diferenças ${ }^{71,72}$.

\section{QUESTÃO: DURANTE A MONITORIZAÇÃO COM O CAP, COM QUE FREQÜÊNCIA MÍNIMA OS PARÂMETROS HEMODINÂMICOS DEVEM SER COLETADOS?}

Recomendação: Pelo menos a cada 4 - 6 horas.

Grau D

Racional: A maioria dos especialistas concorda que sempre que houver alguma intervenção, o resultado deverá ser avaliado em seguida. O grande benefício do suporte hemodinâmico reside em realizar as intervenções precocemente e a monitorização contínua das variáveis hemodinâmicas pode contribuir para a detecção imediata de alterações que as necessitem ${ }^{65}$.

\section{QUESTÃO: E QUEM DEVE COLETAR OS PARÂMETROS HEMODINÂMICOS?}

Recomendação: Médicos e enfermeiros, desde que devidamente treinados.

Grau D
Racional: É fundamental, para a obtenção e utilização aceitáveis das informações fornecidas pelo CAP, que as entidades e sociedades invistam no treinamento dos profissionais médicos e enfermeiros envolvidos no processo $^{73-75}$.

\section{QUESTÃO: COMO AFERIR ADEQUADAMENTE A POAP?}

Recomendação: Tanto sob ventilação com pressão positiva quanto em respiração espontânea, a POAP deve ser aferida na fase expiratória do ciclo respiratório.

Recomendação: Grau C.

Racional: Sob ventilação espontânea: localizar e compor a curva da POAP, antes que as pressões diminuam com a inspiração; sob ventilação mecânica: localizar o componente "a" da curva da POAP antes que as pressões aumentem com a inspiração. Analisar as curvas sempre acompanhadas do ECG, que auxilia na identificação da onda "a", a qual ocorre após o complexo QRS. Nos casos onde não se tem disponível o monitor com capacidade de congelar a imagem, localizar o ponto adequado utilizando réguas vertical e horizontal. Sempre considerar a pressão diastólica da POAP durante a expiração, seja sob VM ou espontânea ${ }^{76-78}$.

\section{QUESTÃO: NA PRÁTICA, OS PROFISSIONAIS DE MEDICINA INTENSIVA TÊM DIFICULDADES NA INTERPRETAÇÃO DOS DADOS OBTIDOS COM O CAP?}

Recomendação: Sim.

Grau B3.

Racional: Existem vários estudos demonstrando falta de precisão das respostas quando profissionais que atuam em UTI são colocados frente a questões sobre a interpretação de dados provenientes do $\mathrm{CAP}^{70,79}$.

\section{QUESTÃO: A VARIABILIDADE NA INTERPRETAÇÃO DOS DADOS OBTIDOS COM O CAP PODERIA DETERMINAR CONDUTAS TERAPÊUTICAS DIFERENTES?}

Recomendação: Sim.

Grau B2

Racional: Os estudos apontam para diferentes opiniões e condutas terapêuticas, a partir dos mesmos dados obtidos e da sua interpretação ${ }^{77,78}$. 


\section{QUESTÃO: A POAP DEVE SER INTERPRETADA DE FORMA INDIVIDUALIZADA EM SITUAÇÕES ESPECÍFICAS?}

Recomendação: A medida da POAP deverá ser interpretada diante da condição clínica do paciente.

Grau C

Racional: A utilização de PEEP pode influenciar a pressão alveolar, a pressão transmural e, por conseguinte, a pressão diastólica do VE. Níveis acima de $12 \mathrm{cmH}_{2} \mathrm{O}$ podem determinar aumento da POAP, sem necessariamente significar aumento da pressão diastólica do VE. Uma abordagem mais prática, no entanto, que evitaria submeter o paciente ao fenômeno colapso/reabertura alveolar, seria considerar apenas a variação da POAP frente às intervenções hemodinâmicas e locar uma curva POAP versus DC. Em outras palavras, o valor exato deveria ser considerado secundário e o comportamento da POAP o principal dado a ser estimado, em resposta a intervenções como a reposição volêmica. Na LPA/SDRA deve-se procurar manter a menor POAP necessária para garantir parâmetros hemodinâmicos e de perfusão tecidual adequados. Na síndrome compartimental abdominal, a elevação da pressão intraabdominal ocasiona o aumento das pressões intratorácicas e a diminuição da complacência pulmonar. Essa condição poderia levar a uma diminuição do retorno venoso e do índice cardíaco e, ainda assim, a medida da POAP ser superestimada. Na disfunção diastólica do ventrículo esquerdo, a relação pressão/volume, que representa a complacência ventricular, encontra-se alterada. Portanto, variações de pressão com volume devem ser interpretadas de maneira especial como representantes de variações no volume diastólico final de VE. Assim, uma POAP mais elevada pode refletir apenas a alteração da complacência, sem significar volemia adequada ou hipervolemia. Nesta situação, construir uma curva POAP x DC, além de revelar a disfunção diastólica, auxilia na titulação da reposição volêmica ${ }^{32,80-82}$.

\section{QUESTÃO: COMO AFERIR ADEQUADAMENTE O DÉBITO CARDÍACO COM CAP UTILIZANDO MEDIDAS INTERMITENTES ATRAVÉS DE BOLUS DE SOLUÇÃO RESFRIADA?}

Recomendação: A diferença entre a temperatura corporal e a do injetado deve ser de pelo menos $12^{\circ} \mathrm{C}$. A velocidade de injeção não interfere, desde que não ultrapasse 4 segundos. Para reduzir a influência da VM com pressão positiva, o termodiluidor deve ser injetado durante a expiração. Deve-se informar ao monitor de débito o volume de solução injetado ( 5 ou $10 \mathrm{~mL}$ ), a temperatura da solução $\left(0-24^{\circ} \mathrm{C}\right)$, o local onde a temperatura da solução está sendo medida e a constante de termodiluição do cateter. Procurar injetar a solução na mesma fase do ciclo respiratório, evitar movimentação excessiva do paciente durante a medida e fazer no mínimo três medidas sem diferença acima de $10 \%$ entre as mesmas. $\mathrm{O}$ uso de sistemas especiais para medida do DC é recomendado para reduzir os fatores de erro ${ }^{32,83}$.

Grau C

Racional: Tais medidas visam a reduzir ao máximo a possibilidade de erro de medida.

\section{QUESTÃO: COMO AVALIAR ADEQUADAMENTE O POSICIONAMENTO DO CAP?}

Recomendação: Através de radiografia de tórax, identificação das ondas de pressão e análise gasométrica. Grau C

Racional: A radiografia de tórax é útil para afastar complicações e determinar a posição do CAP, mas não é muito confiável para determinar o seu posicionamento em zona 3 de West. Na incidência ântero-posterior, a extremidade do CAP deve distar entre 3 a $5 \mathrm{~cm}$ da linha média. Alguns estudos sugerem a necessidade de radiografia lateral em pacientes com níveis elevados de PEEP, para garantir posicionamento abaixo do nível do átrio esquerdo.

A curva da POAP deve ter morfologia adequada, tanto na inspiração como na expiração e seus valores devem ser sempre inferiores à pressão diastólica da artéria pulmonar. A aspiração e análise do sangue colhido com o balonete insuflado confirmam a oclusão de um ramo da artéria pulmonar ${ }^{84}$.

\section{PAINEL DE ESPECIALISTAS DO CONSENSO BRASILEIRO DE MONITORIZAÇÃO E SUPORTE HEMODINÂMICO}

Coordenador: Ederlon Rezende, Diretor do Serviço de Terapia Intensiva do Hospital do Servidor Público Estadual (São Paulo-SP);

Comitê Consultivo: Álvaro Réa-Neto, Professor do Departamento de Clínica Médica da UFPR, Chefe da UTI adulto do Hospital de Clínicas da UFPR, Diretor do CEPETI - Centro de Estudos e Pesquisa em Terapia Intensiva (Curitiba-PR); Ciro Leite Mendes, Coordena- 
dor da UTI adulto do Hospital de Emergência e Trauma Senador Humberto Lucena e Hospital Santa Paula, Médico da Rotina da UTI adulto do Hospital Universitário - UFPB (João Pessoa-PB); Fernando Suparregui Dias, Professor da Faculdade de Medicina da PUC de Porto Alegre, Chefe da UTI geral do Hospital São Lucas da PUC de Porto Alegre (Porto Alegre-RS); GuiIherme Schettino, Doutor em Medicina pela Faculdade de Medicina da USP, Médico Assistente da UTI Respiratória do HC da FMUSP, Médico Coordenador da UTI do Hospital Sírio Libanês (São Paulo-SP); Suzana Margareth Ajeje Lobo, Doutora de Medicina pela Universidade de São Paulo, Professora de clínica médica da Faculdade de Medicina de São José do Rio Preto, Coordenadora do Serviço de Terapia Intensiva do Hospital de Base - FAMERP (São José do Rio Preto-SP); Cid Marcos David, Presidente do Fundo de Educação e Pesquisa da Associação de Medicina Intensiva Brasileira, Doutor em Ciências Médicas, Professor Adjunto da UFRJ (Rio de Janeiro-RJ).

Painel de Especialistas: Alberto Barros, Médico Coordenador da UTI do Hospital Português de Recife (Recife-PE); Eliézer Silva, Doutor em Medicina, Médico Supervisor CTI Hospital Israelita Albert Einstein (São Paulo-SP); Gilberto Friedman, Editor Chefe da Revista Brasileira de Terapia intensiva; Médico do Hospital de Clínicas de Porto Alegre FAMED-UFRGS (Porto AlegreRS); José Luiz Gomes do Amaral, Doutor em Medicina, Chefe da Disciplina de Anestesiologia, Dor e Terapia Intensiva da UNIFESP (São Paulo-SP); Marcelo Park, Médico Assistente da Unidade de Terapia Intensiva Disciplina de Emergências Clínicas - HC-FMUSP (São Paulo-SP); Maristela Monachini, Doutora em Medicina, Médica Assistente da UTI do Hospital Sírio Libanês (São Paulo-SP); Mirella Cristine de Oliveira, Médica Coordenadora do Hospital do Trabalhador (CuritibaPR); Murillo Santucci César Assunção, Médico Coordenador Serviço de Terapia Intensiva do Hospital do Servidor Público Estadual (São Paulo-SP); Nelson Akamine, Médico Supervisor CTI Hospital Israelita Albert Einstein (São Paulo-SP); Patrícia Veiga C Mello, Médica Coordenadora da Disciplina de Emergência da Universidade Estadual do Piauí, Coordenadora da UTI do Hospital de Terapia Intensiva (Terezina-PI); Renata Andréa Pietro Pereira, Enfermeira Chefe do Serviço de Terapia Intensiva do Hospital do Servidor Público Estadual (São Paulo-SP); Rubens Costa Filho, Médico Coordenador CTI do PROCARDÍACO (Rio de Janeiro-RJ); Sebastião Araújo, Médico, Professor Doutor Assistente Departamento de Cirurgia da FCM-UNICAMP (Campi-
nas-SP); Sérgio Félix Pinto, Médico Chefe da UTI do Hospital Universitário da UFMS (Campo Grande-MS); Sérgio Ferreira, Médico Chefe da UTI do Hospital Universitário Getúlio Vargas da UFAM (Manaus-AM); Simone Mattoso Mitushima, Enfermeira da UTI do Hospital Sírio Libanês (São Paulo-SP); Sydney Agareno, Médico Vice-Coordenador da UTI Geral do Hospital Português de Salvador (Salvador-BA); e Yuzeth Nóbrega de Assis Brilhante, Médica Coordenadora da UTI do Hospital da UNIMED João Pessoa (João Pessoa-PB).

\section{REFERÊNCIAS}

1. Nowak RM - Resuscitation of the critically ill in the ED: responses of blood pressure, heart rate, shock index, central venous oxygen saturation, and lactate. Am J Emerg Méd,1996;14:218-225.

2. Assunção MSC, Rezende EAC - Monitorização da PVC e da PAM in Monitorização em UTI. $1^{\text {a }}$ Ed, Rio de Janeiro: Revinter; 2004.

3. Rivers E, Nguyen B, Havstad S et al - Early goal-directed therapy in the treatment of severe sepsis and septic shock. $\mathrm{N}$ Engl J Med, 2001;345:1368-1377.

4. Verweij J, Kester A, Stroes W et al - Comparison of three methods for measuring central venous pressure. Crit Care Med, 1986;14:288-290.

5. Barber CJ - Central venous catheter placement for intravenous digital subtraction angiography: an assessment of technical problems and success rate. Br J Radiol, 1989;62:599-602.

6. Mann RL, Carlon GC, Turnbull AD - Comparison of electronic and manometric central venous pressures. Influence of access route. Crit Care Med, 1981;9:98-100.

7. Darovic GO, Zbilut JP - Fluid-Filled Monitoring Systems. 3rd Ed, Philadelphia: W. B. Saunders Company; 2002.

8. Ahrens TS, Schallom L - Comparison of pulmonary artery and centra venous pressure waveform measurements via digital and graphic measurement methods. Heart Lung, 2001;30:26-38.

9. Knopp R, Dailey $\mathrm{RH}$ - Central venous cannulation and pressure monitoring. JACEP 1977;6:358-366.

10. Lefrant JY, Muller L, De La Coussaye JE et al - Risk factors of failure and immediate complication of subclavian vein catheterization in critically ill patients. Intensive Care Med, 2002;28:1036-1041.

11. Polderman $\mathrm{KH}$, Girbes AJ - Central venous catheter use. Part 1: mechanical complications. Intensive Care Med, 2002;28:1-17.

12. Koh WJ, Suh GY, Han J et al - Recruitment maneuvers attenuate repeated derecruitment-associated lung injury. Crit Care Med, 2005;33:1070-1076.

13. Ruesch S, Walder B, Tramer MR - Complications of central venous catheters: internal jugular versus subclavian access--a systematic review. Crit Care Med, 2002;30:454-460.

14. Courtois M, Fattal PG, Kovacs SJ et al - Anatomically and physiologically based reference level for measurement of intracardiac pressures. Circulation, 1995;92:1994-2000.

15. Carey CF, Courtois M, Barzilai B et al - Correct zero reference leveling for the pulmonary artery catheter. Circulation, 1995;94:(Suppl1):387-388.

16. Cobb DK, High KP, Sawyer RG et al - A controlled trial of scheduled replacement of central venous and pulmonary-artery catheters. $\mathrm{N}$ Engl J Med, 1992;327:1062-1068.

17. Cook D, Randolph A, Kernerman P et al - Central venous catheter replacement strategies: a systematic review of the literature. Crit Care Med, 1997;25:1417-1424.

18. Mermel LA, Farr BM, Sherertz RJ et al - Guidelines for the management of intravascular catheter-related infections. Clin Infect Dis, 2001;32:12491272.

19. McGee WT, Ackerman BL, Rouben LR et al - Accurate placement of central venous catheters: a prospective, randomized, multicenter trial. Crit Care Med, 1993;21:1118-1123.

20. Forrester JS, Diamond G, McHugh TJ et al - Filling pressures in the right and left sides of the heart in acute myocardial infarction. A reappraisal of central-venous-pressure monitoring. N Engl J Med, 1971;285:190-193.

21. Cohn JN - Blood Pressure measurement in shock. Mechanism of innacu- 
racy in auscultatory and palpatory methods. JAMA, 1967;119:118-122.

22. Wilkins RG - Radial artery cannulation and ischaemic damage: a review. Anaesthesia, 1985;40:896-899.

23. Gardner RM - Accuracy and reliability of disposable pressure transducers coupled with modern pressure monitors. Crit Care Med, 1996;24:879-882.

24. Criado FJ - Percutaneous arterial puncture and endoluminal access techniques for peripheral intervention. J Invasive Cardiol, 1999;11:450456.

25. Gardner RM - Direct arterial pressure monitoring. Curr Anaesth Crit Care, 1990;1:239-246.

26. Thomas F, Parker $\mathrm{J}$, Burke $\mathrm{J}$ et al - Prospective randomized evaluation of indwelling radial vs. femoral arterial catheters in high risk critically ill patients. Crit Care Med,1982;10:226.

27. Ejrup B, Fischer B, Wright IS - Clinical evaluation of blood flow to the hand. The false-positive Allen test. Circulation, 1966;33:778-780.

28. Bedford RF - Invasive Blood Pressure Monitoring. 2nd Ed, New York: Churchill Livingstone; 1990.

29. Mueller HS, Chatterjee K, Davis KB et al - ACC expert consensus document. Present use of bedside right heart catheterization in patients with cardiac disease. American College of Cardiology. J Am Coll Cardiol, 1998;32:840-864.

30. Pulmonary Artery Catheter Consensus Conference: consensus statement. New Horiz, 1997;5:175-194.

31. Marini JJ - Acute lung injury. Hemodynamic monitoring with the pulmonary artery catheter. Crit Care Clin, 1986;2:551-572.

32. Perret C, Tagan D, Feihl F et al - The Pulmonary Artery Catheter in Critical Care: a Concise Handbook Cambridge: Blackwell Science Inc, 1996.

33. Ware LB, Matthay MA - The acute respiratory distress syndrome. N Engl J Med, 2000;342:1334-1349.

34. Humphrey H, Hall J, Sznajder I et al - Improved survival in ARDS patients associated with a reduction in pulmonary capillary wedge pressure. Chest, 1990;97:1176-1180.

35. Yu DT, Platt R, Lanken PN et al - Relationship of pulmonary artery catheter use to mortality and resource utilization in patients with severe sepsis. Crit Care Med, 2003;31:2734-2741.

36. Rangel-Frausto MS, Pittet D, Hwang T et al - The dynamics of disease progression in sepsis: Markov modeling describing the natural history and the likely impact of effective antisepsis agents. Clin Infect Dis, 1998;27:185-190.

37. Rangel-Frausto MS, Pittet $\mathrm{D}$, Costigan $\mathrm{M}$ et al - The natural history of the systemic inflammatory response syndrome (SIRS). A prospective study. JAMA, 1995;273:117-123.

38. Vincent JL, De Backer D - Oxygen transport-the oxygen delivery controversy. Intensive Care Med, 2004;30:1990-1996.

39. Reynolds HN, Haupt MT, Thill-Baharozian MC et al - Impact of critical care physician staffing on patients with septic shock in a university hospital medical intensive care unit. JAMA, 1988;260:3446-3450.

40. Ryan TJ, Anderson JL, Antman EM et al - ACC/AHA guidelines for the management of patients with acute myocardial infarction. A report of the American College of Cardiology/American Heart Association Task Force on Practice Guidelines (Committee on Management of Acute Myocardial Infarction). J Am Coll Cardiol, 1996;28:1328-1428.

41. Hasdai D, Topol EJ, Califf RM et al - Cardiogenic shock complicating acute coronary syndromes. Lancet, 2000;356(9231):749-756.

42. Holmes DR Jr, Califf RM, Van de Werf F et al - Difference in countries' use of resources and clinical outcome for patients with cardiogenic shock after myocardial infarction: results from the GUSTO trial. Lancet, 1997;349(9045):75-78.

43. Parrillo JE - Shock: Classification, Pathophysiology and Approach to Management. 2nd Ed, St Louis: Mosby-Year Book; 2001.

44. Mimoz O, Rauss A, Rekik N et al - Pulmonary artery catheterization in critically ill patients: a prospective analysis of outcome changes associated with catheter-prompted changes in therapy. Crit Care Med, 1994;22:573-579.

45. Gilbert WM, Towner DR, Field NT et al - The safety and utility of pulmonary artery catheterization in severe preeclampsia and eclampsia. Am J Obst Gynecol, 2000;182:1397-1403.

46. Kern JW, Shoemaker WC - Meta-analysis of hemodynamic optimization in high-risk patients. Crit Care Med, 2002;30:1686-1692.

47. Boyd O, Grounds RM, Bennett ED - A randomized clinical trial of the effect of deliberate perioperative increase of oxygen delivery on mortality in high-risk surgical patients. JAMA, 1993;270:2699-2707.
48. Berlauk JF, Abrams JH, Gilmour IJ et al - Preoperative optimization of cardiovascular hemodynamics improves outcome in peripheral vascular surgery. A prospective, randomized clinical trial. Ann Surg, 1991;214:289-299.

49. Kasoff SS, Lansen TA, Holder D et al - Aggressive physiologic monitoring of pediatric head trauma patients with elevated intracranial pressure. $\mathrm{Pe}-$ diatr Neurosci, 1988;14:241-249.

50. Rosenwasser RH, Jallo JI, Getch CC et al - Complications of Swan-Ganz catheterization for hemodynamic monitoring in patients with subarachnoid hemorrhage. Neurosurgery, 1995;37:872-876.

51. Costard-Jackle A, Fowler MB - Influence of preoperative pulmonary artery pressure on mortality after heart transplantion: testing of potential reversibility of pulmonary hypertension with nitroprusside is useful in defining a high risk group. J Am Coll Cardiol, 1992:19:48-54.

52. Tuman KJ, McCarthy RJ, Spiess BD et al - Effect of pulmonary artery catheterization on outcome in patients undergoing coronary artery surgery. Anesthesiology, 1989;70:199-206.

53. Pearson KS, Gomez MN, Moyers JR et al - A cost/benefit analysis of randomized invasive monitoring for patients undergoing cardiac surgery. Anesth Analg, 1989;69:336-341.

54. Zaroff JG, Rosengard BR, Armstrong WF et al - Consensus conference report: maximizing use of organs recovered from the cadaver donor: cardiac recommendations, March 28-29, 2001, Crystal City, Va. Circulation, 2002;106:836-841.

55. Van Bakel AB - The cardiac transplant donor: identification, assessment, and management. Am J Med Sci, 1997;314:153-163.

56. Schiller WR, Bay RC, Garren RL et al - Hyperdynamic resuscitation improves survival in patients with life-threatening burns. J Burn Care Rehabil, 1997;18:(1Pt1):10-16.

57. Fleming A, Bishop M, Shoemaker $\mathrm{W}$ et al - Prospective trial of supranormal values as goals of resuscitation in severe trauma. Arch Surg, 1992;127:1175-1181.

58. Bishop MH, Shoemaker WC, Appel PL et al - Prospective, randomized trial of survivor values of cardiac index, oxygen delivery, and oxygen consumption as resuscitation endpoints in severe trauma. J Trauma, 1995;38:780-787.

59. Holm C, Mayr M, Tegeler $\mathrm{J}$ et al - A clinical randomized study on the effects of invasive monitoring on burn shock resuscitation. Burns, 2004;30:798-807

60. Berisha S, Kastrati A, Goda A et al - Optimal value of filling pressure in the right side of the heart in acute right ventricular infarction. Br Heart $\mathrm{J}$, 1990;63:98-102

61. Dell'Italia LJ, Starling MR, Crawford MH et al - Right ventricular infarction: identification by hemodynamic measurements before and after volume loading and correlation with noninvasive techniques. J Am Coll Cardiol, 1984;4:931-939.

62. Parmley W, Chatterjee K - Bedside Hemodynamic Monitoring in Cardiology. Philadelphia: W B Saunders Company, 1992.

63. Sandham JD, Hull RD, Brant RF et al - A randomized, controlled trial of the use of pulmonary-artery catheters in high-risk surgical patients. $\mathrm{N}$ Engl J Med, 2003;348:5-14.

64. Mermel LA, Maki DG - Infectious complications of Swan-Ganz pulmonary artery catheters. Pathogenesis, epidemiology, prevention, and management. Am J Respir Crit Care Med, 1994;149(4Pt1):1020-1036.

65. Chen YY, Yen DH, Yang YG et al - Comparison between replacement at 4 days and 7 days of the infection rate for pulmonary artery catheters in an intensive care unit. Crit Care Med, 2003;31:1353-1358.

66. Nelson LD - The new pulmonary arterial catheters. Right ventricle ejection fraction and continuous cardiac output. Crit Care Clin, 1996;12:795-818.

67. Diebel LN, Wilson RF, Tagett MG et al - End-diastolic volume. A better indicator of preload in the critically ill. Arch Surg, 1992;127:817-822.

68. Cheatham ML, Nelson LD, Chang MC et al - Right ventricular end-diastolic volume index as a predictor of preload status in patients on positive end-expiratory pressure. Crit Care Med, 1998;26:1801-1806.

69. Krafft $\mathrm{P}$, Steltzer $\mathrm{H}$, Hiesmayr $\mathrm{M}$ et al - Mixed venous oxygen saturation in critically ill septic shock patients. The role of defined events. Chest, 1993;103:900-906.

70. Iberti TJ, Fischer EP, Leibowitz AB et al - A multicenter study of physicians' knowledge of the pulmonary artery catheter. Pulmonary Artery Catheter Study Group. JAMA, 1990;264:2928-2932.

71. Connors AF Jr, Speroff T, Dawson NV et al - The effectiveness of right heart catheterization in the initial care of critically ill patients. SUPPORT Investigators. JAMA, 1996;276:889-897. 
72. Richard C, Warszawski J, Anguel $\mathrm{N}$ et al - Early use of the pulmonary artery catheter and outcomes in patients with shock and acute respiratory distress syndrome: a randomized controlled trial. JAMA, 2003;290:2713-2720.

73. Bernard GR, Sopko G, Cerra F et al - Pulmonary artery catheterization and clinical outcomes: National Heart, Lung, and Blood Institute and Food and Drug Administration Workshop Report. Consensus Statement. JAMA, 2000;283:2568-2572.

74. Dietz B, Smith TT - Enhancing the accuracy of hemodynamic monitoring. J Nurs Care Qual, 2002;17:27-34.

75. Druding MC - Integrating hemodynamic monitoring and physical assessment. Dimens Crit Care Nurs, 2000;19:25-30

76. Hoyt JD, Leatherman JW - Interpretation of the pulmonary artery occlusion pressure in mechanically ventilated patients with large respiratory excursions in intrathoracic pressure. Intensive Care Med, 1997;23:1125-1131.

77. Jacka MJ, Cohen MM, To T et al - Pulmonary artery occlusion pressure estimation: how confident are anesthesiologists? Crit Care Med, 2002;30:1197-1203.

78. Komadina $\mathrm{KH}$, Schenk DA, LaVeau $\mathrm{P}$ et al - Interobserver variability in the interpretation of pulmonary artery catheter pressure tracings. Chest, 1991;100:1647-1654.

79. Gnaegi A, Feihl F, Perret C - Intensive care physicians' insufficient knowledge of right-heart catheterization at the bedside: time to act? Crit Care Med, 1997;25:213-220.

80. Pinsky MR - Pulmonary artery occlusion pressure. Intensive Care Med, 2003;29:19-22.

81. Pinsky MR - Clinical significance of pulmonary artery occlusion pressure. Intensive Care Medicine, 2003;29:175-178.

82. Nunes S, Ruokonen E, Takala J - Pulmonary capillary pressures during the acute respiratory distress syndrome. Intensive Care Med, 2003;29:2174-2179.

83. Runciman WB, Ilsley AH, Roberts JG - An evaluation of thermodilution cardiac output measurement using the Swan-Ganz catheter. Anaesth Intensive Care, 1981;9:208-220.

84. Shasby DM, Dauber IM, Pfister S et al - Swan-Ganz catheter location and left atrial pressure determine the accuracy of the wedge pressure when positive end-expiratory pressure is used. Chest, 1981;80:666-670.

Fernando Suparregui Dias, Professor da Faculdade de Medicina da PUC de Porto Alegre, Chefe da UTI geral do Hospital São Lucas da PUC de Porto Alegre (Porto Alegre-RS);

Ederlon Rezende, Diretor do Serviço de Terapia Intensiva do Hospital do Servidor Público Estadual (São Paulo-SP);

Ciro Leite Mendes, Coordenador da UTI adulto do Hospital de Emergência e Trauma Senador Humberto Lucena e Hospital Santa Paula, Médico da Rotina da UTI adulto do Hospital Universitário - UFPB (João Pessoa-PB);

Álvaro Réa-Neto, Professor do Departamento de Clínica Médica da UFPR, Chefe da UTI adulto do Hospital de Clínicas da UFPR, Diretor do CEPETI - Centro de Estudos e Pesquisa em Terapia Intensiva (Curitiba-PR);

Cid Marcos David, Presidente do Fundo de Educação e Pesquisa da Associação de Medicina Intensiva Brasileira, Doutor em Ciências Médicas, Professor Adjunto da UFRJ (Rio de Janeiro-RJ).

Guilherme Schettino, Doutor em Medicina pela Faculdade de Medicina da USP, Médico Assistente da UTI Respiratória do HC da FMUSP, Médico Coordenador da UTI do Hospital Sírio Libanês (São Paulo-SP);
Suzana Margareth Ajeje Lobo, Doutora de Medicina pela Universidade de São Paulo, Professora de clínica médica da Faculdade de Medicina de São José do Rio Preto, Coordenadora do Serviço de Terapia Intensiva do Hospital de Base - FAMERP (São José do Rio Preto-SP); Alberto Barros, Médico Coordenador da UTI do Hospital Português de Recife (Recife-PE);

Eliézer Silva, Doutor em Medicina, Médico Supervisor CTI Hospital Israelita Albert Einstein (São Paulo-SP);

Gilberto Friedman, Editor Chefe da Revista Brasileira de Terapia intensiva; Médico do Hospital de Clínicas de Porto Alegre FAMED-UFRGS (Porto Alegre-RS);

José Luiz Gomes do Amaral, Doutor em Medicina, Chefe da Disciplina de Anestesiologia, Dor e Terapia Intensiva da UNIFESP (São Paulo-SP);

Marcelo Park, Médico Assistente da Unidade de Terapia Intensiva - Disciplina de Emergências Clínicas - HC-FMUSP (São Paulo-SP);

Maristela Monachini, Doutora em Medicina, Médica Assistente da UTI do Hospital Sírio Libanês (São Paulo-SP); Mirella Cristine de Oliveira, Médica Coordenadora do Hospital do Trabalhador (Curitiba-PR);

Murillo Santucci César Assunção, Médico Asistente da Disciplina de Anestesiologia, Dor e Terapia Intensiva da UNIFESP (São Paulo-SP);

Nelson Akamine, Médico Supervisor CTI Hospital Israelita Albert Einstein (São Paulo-SP);

Patrícia Veiga C Mello, Médica Coordenadora da Disciplina de Emergência da Universidade Estadual do Piauí, Coordenadora da UTI do Hospital de Terapia Intensiva (Terezina-PI);

Renata Andréa Pietro Pereira, Enfermeira Chefe do Serviço de Terapia Intensiva do Hospital do Servidor Público Estadual (São Paulo-SP);

Rubens Costa Filho, Médico Coordenador CTI do PROCARDÍACO (Rio de Janeiro-RJ);

Sebastião Araújo, Médico, Professor Doutor Assistente Departamento de Cirurgia da FCM-UNICAMP (Campinas-SP);

Sérgio Félix Pinto, Médico Chefe da UTI do Hospital Universitário da UFMS (Campo Grande-MS);

Sérgio Ferreira, Médico Chefe da UTI do Hospital Universitário Getúlio Vargas da UFAM (Manaus-AM);

Simone Mattoso Mitushima, Enfermeira da UTI do Hospital Sírio Libanês (São Paulo-SP);

Sydney Agareno, Médico Vice-Coordenador da UTI Geral do Hospital Português de Salvador (Salvador-BA); Yuzeth Nóbrega de Assis Brilhante, Médica Coordenadora da UTI do Hospital da UNIMED João Pessoa (João Pessoa-PB). 